Note: This copy is for your personal non-commercial use only. To order presentation-ready copies for distribution to your colleagues or clients, contact us at www.rsna.org/rsnarights.

\title{
Multicenter Comparative Multimodality Surveillance of Women at Genetic-Familial High Risk for Breast Cancer (HIBCRIT Study): Interim Results ${ }^{1}$
}

Francesco Sardanelli, MD

Franca Podo, DrSci

Giuliano D'Agnolo, PhD

Arduino Verdecchia, DrSci

Mariano Santaquilani, Tech Eng

Renato Musumeci, MD

Giovanna Trecate, MD

Siranoush Manoukian, MD

Sandro Morassut, MD

Clelia de Giacomi, MD

Massimo Federico, MD

Laura Cortesi, MD

Stefano Corcione, MD

Stefano Cirillo, MD

Vincenzo Marra, MD

For the High Breast Cancer Risk Italian Trial (HIBCRIT)

\footnotetext{
${ }^{1}$ From the University of Milan School of Medicine, Department of Medical and Surgical Sciences, Unit of Radiology, IRCCS Policlinico San Donato, Via Morandi 30, 20097 San Donato Milanese, Milan, Italy (F.S.); and Department of Cell Biology and Neurosciences, Istituto Superiore di Sanità, Rome, Italy (F.P.). The remaining authors and their affiliations are listed at the end of this article. From the 2004 RSNA Annual Meeting. Received December 5, 2005; revision requested January 25, 2006; revision received February 28; accepted April 3; final version accepted June 30 . The HIBCRIT study was supported by the Italian Ministry of Health (Ricerca Finalizzata 1\% 98/JT/T) and Istituto Superiore di Sanità (ricerca corrente C3A3/2004) and coordinated by the Istituto Superiore di Sanità, Rome, Italy. Address correspondence to F.S. (e-mail: f.sardanelli@grupposandonato.it).
}

๑ RSNA, 2007
Purpose:

Materials and Methods:

Results:

\section{Conclusion:}

To prospectively compare clinical breast examination (CBE), mammography, ultrasonography (US), and contrast material-enhanced magnetic resonance (MR) imaging for screening women at genetic-familial high risk for breast cancer and report interim results, with pathologic findings as standard.

Institutional review board of each center approved the research; informed written consent was obtained. CBE, mammography, US, and MR imaging were performed for yearly screening of BRCA1 or BRCA2 mutation carriers, first-degree relatives of BRCA1 or BRCA2 mutation carriers, or women enrolled because of a strong family history of breast or ovarian cancer (three or more events in firstor second-degree relatives in either maternal or paternal line; these included breast cancer in women younger than 60 years, ovarian cancer at any age, and male breast cancer at any age).

Two hundred seventy-eight women (mean age, 46 years \pm 12 [standard deviation]) were enrolled. Breast cancer was found in 11 of 278 women at first round and seven of 99 at second round (14 invasive, four intraductal; eight were $\leq 10 \mathrm{~mm}$ in diameter). Detection rate per year was $4.8 \%$ (18 of 377) overall; $4.3 \%$ (11 of 258) in BRCA1 or BRCA2 mutation carriers and first-degree relatives of BRCA1 or BRCA2 mutation carriers versus 5.9\% (seven of 119) in women enrolled because of strong family history; and $5.3 \%$ (nine of 169) in women with previous personal breast and/or ovarian cancer versus $4.3 \%$ (nine of 208) in those without. In six (33\%) of 18 patients, cancer was detected only with MR imaging. Sensitivity was as follows: CBE, 50\% (95\% confidence interval [CI]: 29\%, 71\%); mammography, 59\% (95\% CI: 36\%, 78\%); US, 65\% (95\% CI: $41 \%, 83 \%$ ); and MR imaging, 94\% (95\% CI: $82 \%$, 99\%). Positive predictive value was as follows: CBE, $82 \%$ (95\% CI: 52\%, 95\%); mammography, 77\% (95\% CI: 50\%, $92 \%)$; US, $65 \%$ (95\% CI: 41\%, 83\%); and MR imaging, $63 \%$ (95\% CI: 43\%, 79\%).

Addition of MR imaging to the screening regimen for highrisk women may enable detection of otherwise unsuspected breast cancers.

○ RSNA, 2007 
he cumulative lifetime risk of breast cancer is greatly different for women with a hereditary predisposition for this disease from that for women in the general population. Although breast cancer affects a range of as many as one in seven to one in 11 women in Western countries $(1,2)$, sporadic disease accounts for about $85 \%-$ $95 \%$ of the occurrences. The remaining $5 \%-15 \%$ of breast cancers are clustered in families with high breast cancer incidence (3-5). For women in the latter group, an inherited predisposition may result in an early onset of the disease (typically in the premenopausal period) and in a lifetime risk greater than $50 \%-60 \%(3-7)$.

\section{Advances in Knowledge}

- By using a multimodality surveillance approach that included contrast-enhanced MR imaging, the detection rate of breast cancer in asymptomatic high-risk women was $4.8 \%$ in our study.

- In women enrolled only on the basis of well-defined criteria for strong family history of breast and/or ovarian cancer, the detection rate was not significantly different from that of those enrolled for positive BRCA1 or BRCA2 genetic testing (or for being firstdegree relatives of subjects with positive BRCA1 or BRCA2 genetic testing).

- Contrast-enhanced MR imaging showed a high sensitivity $(94 \%$; $33 \%$ of patients with cancer detected only with contrast-enhanced MR imaging), with an acceptable positive predictive value $(63 \%)$ that was close to that of US (65\%); the addition of contrastenhanced MR imaging to the screening regimen of high-risk women may enable the detection of otherwise unsuspected breast cancers.

- Contrast-enhanced MR imaging showed a high sensitivity in the detection of multiple malignant foci in the same breast in highrisk women with invasive cancers.
About $50 \%$ of the occurrences in high-risk families can be explained by the dominant autosomal inheritance of the deleterious BRCA1 or BRCA2 mutations on the $17 q 21$ or the $13 q 12$ chromosomal location, respectively, with incomplete penetrance $(6,8,9)$. BRCA1 mutations are associated with an increased risk of ovarian cancer, whereas BRCA2 mutations may be associated with male breast cancer. Breast cancers in very young women (younger than 35 years) are frequently associated with BRCA1 mutations (10). Moreover, breast cancers in BRCA1 mutation carriers are more frequently high grade and estrogen receptor negative compared with sporadic cancers $(11,12)$, and they are associated with less favorable survival $(13,14)$.

The strategy for surveillance of high-risk women is different from that for surveillance of the general population (eg, annual mammography for women older than 40 years in the United States and biannual mammography for women older than 50 years in many European countries). Screening for high-risk women should begin at a younger age and with no more than a 1-year interval between examinations. As reported in a recent review (15), researchers determined that contrast material-enhanced magnetic resonance (MR) imaging is highly sensitive for identification of breast cancer in a large spectrum of clinical situations. Because of lower values for specificity and positive predictive value (PPV), with contrast-enhanced MR imaging, however, unnecessary breast biopsy has been reported because of false-positive findings $(16,17)$, especially in premenopausal women $(18,19)$ and also in high-risk women (20).

Findings from two retrospective $(21,22)$ and five prospective studies (23-27) indicated that contrast-enhanced MR imaging is useful in the screening of high-risk women. These single- and multicenter studies differed in the number of diagnostic modalities used for comparative evaluation, in enrollment criteria, in selected age groups, and in levels of cumulative lifetime risk of breast cancer. The purpose of our mul- ticenter trial was to prospectively compare the sensitivity of clinical breast examination (CBE), mammography, ultrasonography (US), and contrast-enhanced MR imaging for the screening of women at genetic-familial high risk for breast cancer and to report our interim results, with pathologic findings as the reference standard.

\section{Materials and Methods}

\section{Study Design and Population}

An open prospective nonrandomized multicenter comparative trial was designed (7), and the institutional review board of each center approved the research. Informed written consent was obtained from each subject enrolled in the study. We planned to enroll asymptomatic subjects at high risk for breast cancer, women at least 25 years of age and men at least 50 years of age, who were (a) carriers of BRCA1 or BRCA2 mutations, as demonstrated with genetic testing, or subjects with personal unknown mutational status but who were first-degree relatives of BRCA1 or BRCA2 mutation carriers or (b) subjects with a strong family history of breast or ovarian cancer who had three or more events of breast or ovarian cancer in first- or second-degree relatives in either the maternal or the paternal line. These three or more events could have included female breast cancer in relatives younger than 60 years, ovarian cancer in relatives at any age, or male breast cancer in relatives at any age.

The occurrence of previous breast cancer and/or ovarian cancer in the

\section{Published online before print 10.1148/radiol.2423051965}

\section{Radiology 2007; 242:698-715}

\section{Abbreviations:}

BI-RADS = Breast Imaging Reporting and Data System $\mathrm{CBE}=$ clinical breast examination

PPV $=$ positive predictive value

\section{Author contributions:}

The list of author contributions is at the end of this article.

Authors stated no financial relationship to disclose. 
subject to be enrolled could contribute to the number of events needed to meet the second criterion defined previously. A multifocal unilateral cancer was regarded as one event. A multicentric unilateral cancer was regarded as two events. An ipsilateral metachronous breast cancer was regarded as a new event if it was located at least at $5 \mathrm{~cm}$ from the previous cancer. Bilateral synchronous breast cancers were regarded as two different events.

Subjects with a history of personal breast cancer were included (28), provided that at least one breast was not completely excised. Exclusion criteria were pregnancy, breast-feeding, current chemotherapy, terminal illness, and contraindications to MR imaging. History of surveillance imaging prior to entry into the study was not specifically recorded. For each enrolled patient, we planned two annual rounds of assessment with $\mathrm{CBE}$, mammography, US, and contrast-enhanced MR imaging and at least a 1-year follow-up with CBE, mammography, and US. At each screening event, each of these tests was performed by an independent physician who was unaware of the results obtained by the other three colleagues.

Centers were asked to plan the independent examinations for each screening event in the same day for as many enrolled subjects as possible. When we considered the need for planning contrast-enhanced MR imaging during the 2nd week of the menstrual cycle in premenopausal women and logistic problems (eg, different locations for mammography, US, and MR imaging facilities; members of the same family preferred to have the examination on the same day), a maximum time of 1 month for performance of the four diagnostic modalities was allowed. In some instances, justified by particular conditions, the central unit accepted a maximum delay of 2 months.

A national health institution (Istituto Superiore di Sanità, Rome, Italy) was responsible for multicenter project design, central coordination, and data management and funding (central unit). This institution was not directly involved in the enrollment or in the per- formance of genetic testing, CBE, mammography, breast US, and contrast-enhanced MR imaging. Seventeen centers in 14 Italian towns participated. The first center was activated in June 2000, and the 17 th center was activated in November 2003; four institutes for cancer research and treatment, 12 university hospitals, and one general hospital were included. Quality assessment programs were regularly performed in the radiology departments of these institutions. At each center, examinations were performed, and findings from the examinations were interpreted with the supervision of a radiologist who had at least 10 years of experience in breast imaging; moreover, other physicians were involved in the trial at each center, and the centers and names of those involved appear at the end of this article.

During the first phase of the study (from June 2000 to December 2002), the enrollment was limited to the first criterion described before; preliminary results for this phase, which pertained to the first eight patients in whom breast cancer was detected, were reported during an international workshop in early 2002 (7). In January 2003, enrollment on the basis of the second criterion was also opened. The general plan of the trial was aimed at enrolling approximately 500 subjects in about 6 years, with about $50 \%$ fulfilling the first criterion and about 50\% fulfilling the second criterion. For each of the three diagnostic imaging modalities (mammography, US, and contrast-enhanced MR imaging), performed in nonrandomized order, the results of local prospective independent readings are included in the data presented here. Each reader was aware of the high-risk condition of the women but was blinded to the results of the other three diagnostic modalities. During the second round, the readers were aware of the results from the first annual round.

\section{Genetic Testing}

Genetic testing for identification of deleterious BRCA1 and BRCA2 mutations included the use of individual or com- bined procedures, such as direct sequencing (29-31), protein truncation testing (29), single-strand conformation polymorphism (32), or mutation screening with denaturing chromatography (33).

\section{Clinical Breast Examination}

Thirty-nine physicians performed CBE in the 17 centers. Findings of each examination were coded as normal, suggestive of benign disease, indeterminate, suspicious for malignant disease, or highly suggestive of malignant disease. Palpable nodules that were judged as benign or indeterminate but were clearly benign at mammography and/or US (eg, cysts, calcified fibroadenomas) were not considered in the current report. In the present analysis, for each patient who had cancer during the trial, the cancer was considered palpable if at least one of the pathologically demonstrated malignant foci was coded at CBE as indeterminate, suspicious for malignant disease, or highly suggestive of malignant disease. Clinical findings were marked on a diagram and correlated with imaging findings after reporting.

\section{Mammographic Examination}

Bilateral two-view mammography (unilateral in women who previously had undergone one-breast mastectomy) was performed with screen-film units with a rotating anode, a $0.3-0.1-\mathrm{mm}$ focus, a focus-film distance of $55 \mathrm{~cm}$ or greater, homogeneous breast compression, a mobile grid, and automatic exposure control. Dedicated daylight processing was performed. Standard mediolateral oblique and craniocaudal projections were acquired. Further dedicated mammograms (ie, magnification, spot compression, or other additional views) were obtained when necessary.

When an enrolled subject underwent a previous mammographic examination performed within the 2 months preceding enrollment, the mammogram from that examination was acquired as part of the study after a quality check; if mammography was performed more than 2 months before the enrollment, the first round of complete multimodal- 
ity assessment was planned 12 months after the previous mammogram was obtained.

Images from mammographic examinations were evaluated by one reader. Evaluations were performed by radiologists with at least 10 years of experience in breast imaging, and findings were classified by using the five-point scale of the Breast Imaging Reporting and Data System (BI-RADS) (34). Thirty-nine radiologists interpreted the mammograms in the 17 centers. The density pattern was classified according to two categories: breasts with more than $50 \%$ of the breast occupied by fibroglandular density as a mean of the two mammographic views and breasts with $50 \%$ or less of the breast occupied by fibroglandular density as a mean of the two mammographic views.

\section{US Examination}

US examinations were performed by radiologists with at least 10 years of experience in breast imaging. Both breasts were systematically examined by using vertical, horizontal, radial, and antiradial scans obtained by a radiologist who reported the findings of the examination by using the five-point BI-RADS scale (35). Forty-one physicians performed and interpreted the US breast examinations in the 17 centers. Transducers with a frequency of $10 \mathrm{MHz}$ or greater

\section{Table 1}

\section{Scoring System with Combination of Morphologic and Dynamic Parameters for Evaluation of Breast Lesions on Contrast-enhanced MR Images}

Criterion

Shape

$\begin{array}{ll}\text { Round, oval, lobular } & 0 \\ \text { Linear, ductal, segmental, dendritic, spiculated } & 1\end{array}$

Margins

Regular $\quad 0$

Irregular

1

Kinetic pattern

Initial enhancement*

$<50 \%$

$\geq 50$ and $<100 \%$

$\geq 100 \%$

Postinitial enhancement

Continuous $^{\dagger}$

Plateau $^{\ddagger}$

Washout $^{\S}$

Enhancement pattern

Homogeneous

Inhomogeneous

Rim

1

(1)

(1)

0
1
2
0
1
2
0
1
2

Source.-References 38, 39.

Note.-Total score interpretation and translation into BI-RADS categories 1-5 is as follows: no detectable enhancement $=$ no enhancing focus $=$ BI-RADS 1 , score $0-2=$ benign $=$ BI-RADS 2 , score $3=$ probably benign $=$ BI-RADS 3 , score $4-5=$ suspicious $=\mathrm{BI}-\mathrm{RADS} 4$, and score $6-8=$ highly suggestive of malignancy $=$ BI-RADS 5 .

* Initial peak signal intensity within the first 3 minutes after contrast agent administration, relative to the signal intensity at unenhanced imaging.

${ }^{\dagger}$ Continuous signal intensity is defined as when a progressive signal intensity increase is found during the 4th, 5th, and 6th minutes and the signal intensity at the 6 th minute is greater than $10 \%$ of that of the initial peak signal intensity obtained during the 1st, 2nd, and 3rd minutes.

${ }^{\ddagger}$ Plateau is defined as when the postinitial signal intensity during the 4th, 5th, and 6th minutes remains within the range of $\pm 10 \%$ of that of the initial peak signal intensity obtained during the $1 \mathrm{st}, 2 \mathrm{nd}$, and 3rd minutes.

$\S$ Washout is defined as when a progressive signal intensity decrease is found during the 4th, 5th, and 6th minutes and the signal intensity at the 6 th minute is less than $10 \%$ of that of the initial peak signal intensity obtained during the 1 st, 2 nd, and 3rd minutes. were used in 14 centers, transducers with a frequency of $7.5 \mathrm{MHz}$ or greater were used in two centers, and only a transducer with a frequency of $7.5 \mathrm{MHz}$ was used in one center; transverse resolution of $0.5 \mathrm{~mm}$ or less and lateraltransverse resolution of $1 \mathrm{~mm}$ or less were used.

\section{Contrast-enhanced MR Imaging}

Contrast-enhanced MR imaging was performed at $1.5 \mathrm{~T}$ (12 centers) or $1.0 \mathrm{~T}$ (four centers), whereas at one center both field strengths were used because of the change of equipment during the trial. The MR units, equipped with actively shielded gradients of $15 \mathrm{mT} / \mathrm{m}$ or greater, were purchased from three manufacturers (GE Medical Systems, Milwaukee, Wis; Philips Medical Systems, Best, the Netherlands; or Siemens Medical Solutions, Erlangen, Germany). Dedicated synchronous breast coils were used for bilateral studies (or unilateral studies when a mastectomy previously had been performed) with the patient in the prone position. The examination was planned on the 7 th14th day of the menstrual cycle in premenopausal women but without scheduling limitations in postmenopausal women.

A long venous catheter access was obtained by using a plastic cannula in a cubital vein. After localizing scout views were obtained, a contrast-enhanced dynamic three-dimensional T1-weighted spoiled gradient-echo sequence was performed in the transverse or coronal plane. The parameters were as follows: repetition time, $13 \mathrm{msec}$ or less; flip angle, $20^{\circ}-30^{\circ}$; partition thickness, 3 $\mathrm{mm}$ or smaller; intersection gap, none; number of partitions, 40-128 (to cover both breasts entirely); and acquisition time, not longer than 120 seconds. The echo time was chosen to prevent fatwater signal opposition at 1.0 or $1.5 \mathrm{~T}$ (36). The field of view and image matrix were combined to obtain a pixel size of $1.4 \times 1.4 \mathrm{~mm}$ or less.

An unenhanced sequence was performed before contrast agent injection. Then, $0.1 \mathrm{mmol} / \mathrm{kg}$ of one of the commercially available $0.5 \mathrm{~mol} / \mathrm{L}$ two- 
compartment gadolinium chelates (gadopentetate dimeglumine, Magnevist, Schering, Berlin, Germany; gadoterate meglumine, Dotarem, Guerbet, Paris, France; gadodiamide, Omniscan, Amersham Health, Oslo, Norway; or gadoteridol, ProHance, Bracco, Milan, Italy) was intravenously administered at the rate of $2 \mathrm{~mL} / \mathrm{sec}$ by using an automatic injector. Injection was followed by flushing with $20 \mathrm{~mL}$ of saline. Taking into account that the central lines of the $\mathrm{k}$ space, mainly devoted to image contrast resolution, were acquired in the central third of the sequence time duration, the contrast agent injection was started at the same time as the first contrast-enhanced sequence was started. The number of contrast-enhanced sequences, typically five with a temporal resolution of 90 seconds, was sufficient to obtain dynamic information during at least the first 6 minutes after contrast agent injection.

Temporal subtraction (enhanced minus unenhanced images) was always performed for the first, second, and last contrast-enhanced sequences, and a maximum intensity projection algorithm was applied for the first and the second contrast-enhanced sequences. Signal intensity-time and percentage of enhancement-time dynamic curves were obtained for targeted small regions of interest positioned on the areas of homogeneous maximal enhancement within the enhancing lesion (37). Source and processed images were electronically stored on compact discs. Twenty-eight radiologists interpreted the contrast-enhanced MR images from breast examinations in the 17 centers.

Morphologic and dynamic parameters were combined according to the criteria first defined by Fischer et al (38) and tested by Baum et al (39) in 1031 breasts. In the study by Baum et al, sensitivity and specificity were both 92\%. This eight-level scoring system was modified into a five-point scale according to the BI-RADS (40) classification (Table 1).

\section{Work-up of Lesions Suspicious for Malignancy and Pathologic Standard of Reference}

An integration across the modalities was performed at each center after each physician had reported findings for CBE, mammography, US, and contrastenhanced MR imaging. In patients who had a palpable breast nodule that was indeterminate or suspicious for malignancy or an imaging-detected lesion classified as BI-RADS 4 or 5 with one or more than one of the three modalities, fine-needle aspiration or core-needle (14-gauge) biopsy was performed. When the lesion was detectable at US (at the first look before contrast-enhanced MR imaging or at the second look after contrast-enhanced MR imaging), the biopsy was performed with US guidance. When the lesion was mammographically detectable and not detectable at US, the biopsy was performed with stereotactic guidance. When the lesion was detectable at only contrast-enhanced MR imaging (which included a

\section{Table 2}

\section{Multimodality Surveillance of 278 Women at High Risk of Breast Cancer: Enrollment and Detection Rate per Year}

\begin{tabular}{lcc} 
Population Characteristics & Enrolled & Detection Rate per Year \\
\hline Enrollment & $98(35.3)$ & $7 / 142(4.9)^{\dagger}$ \\
\hline BRCA1 mutation carriers & $68(24.5)$ & $4 / 103(3.9)^{\dagger}$ \\
BRCA2 mutation carriers & $9(3.2)$ & $0 / 13(0)$ \\
\hline First-degree relatives of BRCA1 or BRCA2 mutation carriers & $175(62.9)$ & $11 / 258(4.3)^{\ddagger}$ \\
Overall no. of BRCA1 or BRCA2 mutation carriers and first-degree relatives of BRCA1 or BRCA2 mutation carriers & $103(37.1)$ & $7 / 119(5.9)^{\ddagger}$ \\
Strong family history of breast cancer and/or ovarian cancer & $278(100)$ & $18 / 377(4.8)$ \\
Overall no. of women who underwent screening & $123(44.2)$ & $9 / 169(5.3)^{\sharp}$ \\
\hline Previous vs no previous breast cancer and/or ovarian cancer & $155(55.8)$ & $9 / 208(4.3)^{\#}$
\end{tabular}

Note.-Data are numbers of women, and numbers in parentheses are percentages. All 278 enrolled women underwent first-round multimodality evaluation, whereas only 99 of them underwent second-round evaluation (44 BRCA1 mutation carriers, 35 BRCA2 mutation carriers, four first-degree relatives of a BRCA1 mutation carrier, no first-degree relatives of a BRCA2 mutation carrier, and 16 women enrolled on the basis of a strong family history of breast cancer and/or ovarian cancer).

* The detection rate per year was calculated for each subset and overall as the ratio between the number of patients who were affected by breast cancer during the first or second round and the number of women who underwent screening at the first round plus the number of women who underwent screening at the second round.

† The difference in the detection rate between BRCA1 mutation carriers and BRCA2 mutation carriers was not significant ( $\chi^{2}$ test).

₹ The difference in the detection rate between BRCA1 or BRCA2 mutation carriers or first-degree relatives of a BRCA1 or BRCA2 mutation carrier and women enrolled on the basis of a strong family history of breast cancer and/or ovarian cancer was not significant $\left(\chi^{2}\right.$ test).

$\S$ Subjects with a strong family history of breast cancer and/or ovarian cancer had first- or second-degree relatives in either the maternal or the paternal line who had at least three female breast cancers in those younger than 60 years, at least three female breast cancers in those younger than 60 years and/or ovarian cancer at any age, or at least three female breast cancers in those younger than 60 years and/or male breast cancer at any age.

" 0 f 123 enrolled patients with previous breast cancer and/or ovarian cancer, 104 had a previous breast cancer, 14 had a previous ovarian cancer, and five had a previous breast cancer and ovarian cancer; seven of these patients with previous breast and/or ovarian cancer had had an inconclusive genetic test (ie, negative for BRCA1 or BRCA2 mutations [BRCAX status]).

\# The difference in the detection rate between women with a previous personal breast cancer and/or ovarian cancer versus women without a previous personal breast cancer and/or ovarian cancer was not significant $\left(\chi^{2}\right.$ test). 


\section{Figure 1}

\begin{tabular}{|c|c|c|}
\hline \multicolumn{2}{|c|}{278 enrolled women at high risk of breast cancer } & \multirow{5}{*}{$\begin{array}{l}\text { Figure 1: Flow diagram of the } \\
\text { multicenter surveillance of women } \\
\text { at high risk for breast cancer by } \\
\text { using contrast-enhanced MR } \\
\text { imaging, mammography, US, and } \\
\text { CBE. }\end{array}$} \\
\hline $\begin{array}{l}278 \text { women underwent } \\
\text { the first round }\end{array}$ & $\begin{array}{l}99 \text { women underwent } \\
\text { the second round }\end{array}$ & \\
\hline $\begin{array}{c}254 \text { negative } \\
\text { screening events }\end{array}$ & $\begin{array}{c}90 \text { negative } \\
\text { screening events }\end{array}$ & \\
\hline $\begin{array}{l}13 \text { invasive procedures for } \\
\text { false positive findings }\end{array}$ & $\begin{array}{l}2 \text { invasive procedures for } \\
\text { false positive findings }\end{array}$ & \\
\hline $\begin{array}{l}11 \text { women found to be } \\
\text { affected with breast cancer }\end{array}$ & $\begin{array}{l}7 \text { women found to be } \\
\text { affected with breast cancer }\end{array}$ & \\
\hline
\end{tabular}

second-look US examination with a negative image), biopsy and presurgical localization were performed with contrast-enhanced MR imaging guidance.

For any finding classified as BIRADS 3 (probably benign) at each imaging modality without a higher classification (ie, BI-RADS 4 or 5) at other diagnostic modalities in the same round, a short-term (within 4 months) follow-up with the same modality with which the lesion was detected was planned. If the finding was confirmed (ie, a lesion with at least a BI-RADS 3 classification and was not reduced in diameter), an imaging-guided biopsy was performed according to the protocol defined previously. When the report for the fine-needle or core biopsy indicated that the lesion was suspicious for malignant disease or highly suggestive of malignancy, the patient always underwent surgery. When the findings in the report for the fine-needle or core biopsy suggested a diagnosis of lobular neoplasia (lobular carcinoma in situ), atypical ductal hyperplasia, radial scar, papilloma, or papillomatosis, the patient underwent surgery, and pathologic examination of the surgical specimen was performed. For all the lesions classified as BI-RADS 4 or 5 at any imaging modality, the patient underwent surgery, and pathologic examination was performed, even though the fine-needle or core biopsy yielded a negative, benign, or probably benign result.

All nonpalpable lesions were preoperatively localized by using a hook wire or a charcoal suspension, with the guid- ance of the imaging method used for biopsy.

At the 17 centers, 19 pathologists who had 9-35 years of experience with breast pathology classified the pathologic specimens according to the 1981 World Health Organization breast cancer classification (41). The maximal diameter of each malignant lesion was recorded. For invasive malignant lesions, axillary nodal status was explored with axillary dissection or sentinel lymph node biopsy, depending on the preference of each center involved in the study.

\section{Statistical Analysis}

Overall detection rate per year was calculated as the ratio between the number of patients with pathologically proved breast cancer and the sum of screening events at the first and the second rounds $(278+99=377)$. The detection rate for each round was calculated as the ratio between the number of patients with pathologically proved breast cancer at each round and the number of women who underwent screening in the same round. The detection rate per year for subsets of enrolled women was calculated as the ratio between the number of patients who had a pathologically proved breast cancer in the subset of women and the number of screening events in the members of the subset of women in the analysis.

Sensitivity of each of the four modalities was calculated as the ratio between the number of true-positive findings (pathologically proved breast cancer detected by using each modality) and the number of true-positive findings plus false-negative findings (pathologically proved breast cancer examined with the same modality but not correctly diagnosed [ie, BI-RADS categories 1-3]). For each modality, the false-negative findings were defined as breast cancers detected by using one, two, or three of the remaining modalities and confirmed at pathologic examination.

PPV of each of the four modalities was calculated as the ratio between true-positive findings (defined as previously mentioned) and the number of true-positive findings plus false-positive findings (findings that needed to be explored with invasive procedures that defined a benign cytologic or pathologic diagnosis). Invasive procedures included fine-needle aspiration, core-needle biopsy, and surgical biopsy after imaging-guided localization (one patient opted for bilateral prophylactic mastectomy, which revealed a mammographic finding as false-positive).

In the evaluation of multiple malignant foci in the same breast, the total number of malignant foci demonstrated at pathologic examination was used as the standard of reference. The capability of each modality for depiction of multiple malignant foci was evaluated without considering the difference between multifocal and multicentric cancers.

For both sensitivity and PPV, 95\% confidence intervals were calculated from the exact binomial distribution.

Differences in the mean age of the enrolled women or patients and in the mean pathologically determined diameter of the tumors were tested by using the two-tailed Mann-Whitney $U$ test. Differences in detection rate, percentage of patients with invasive cancer, and percentage of patients with axillary nodal metastatic involvement were tested by using the $\chi^{2}$ test or the Fisher exact test, when the former was not applicable-that is, for small samples (fewer than 20 subjects sampled)- or when one or more of the so-called expected values was smaller than five (42). Statistical calculations were performed by using a software package (SPSS, version 6.0; SPSS, Chicago, Ill). 


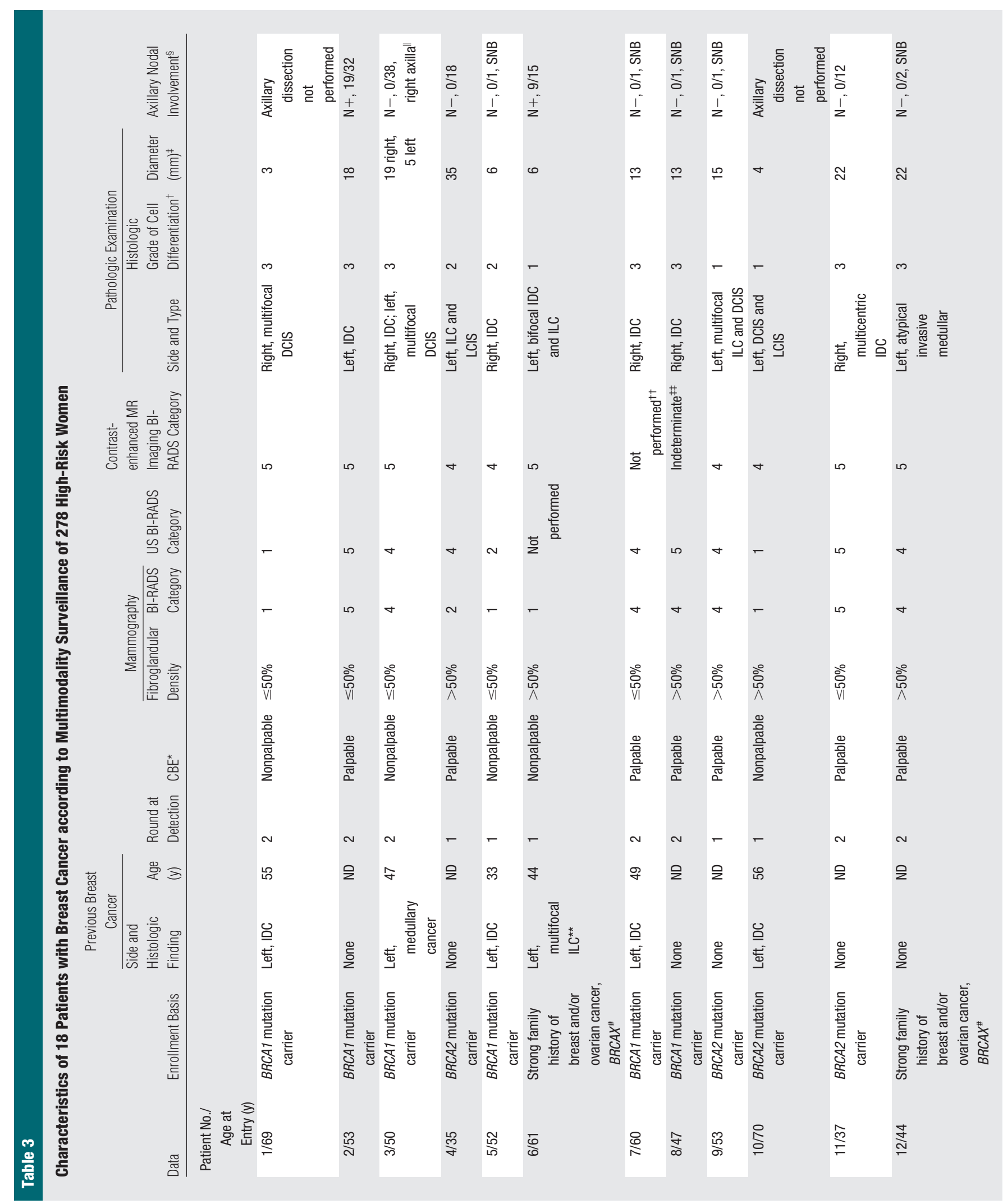




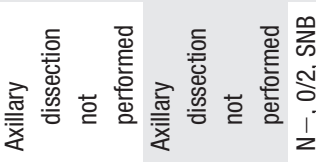
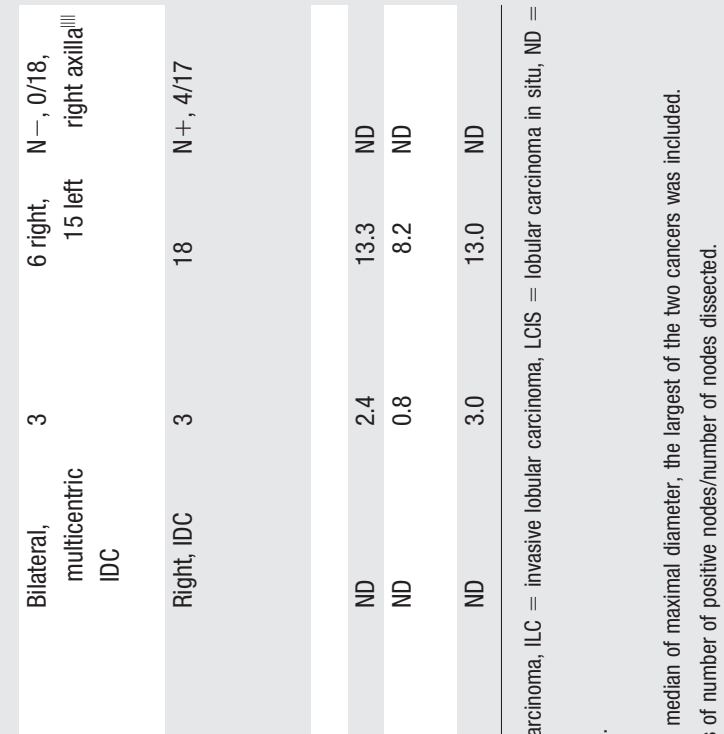

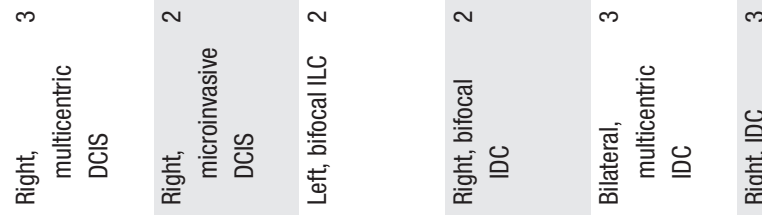

守 $\stackrel{0}{\circ}$ 穴

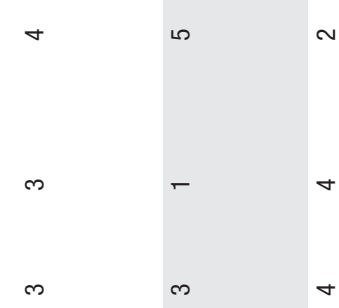

今े

亏ें

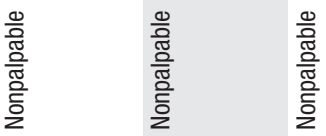

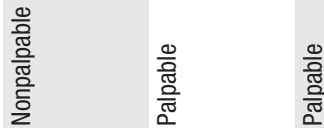

₹

焉

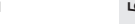

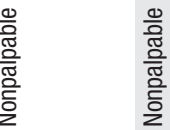

$\frac{\sqrt{2}}{\frac{\pi}{20}}$

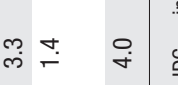

लำ

$\stackrel{+}{\circ}$

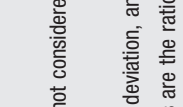
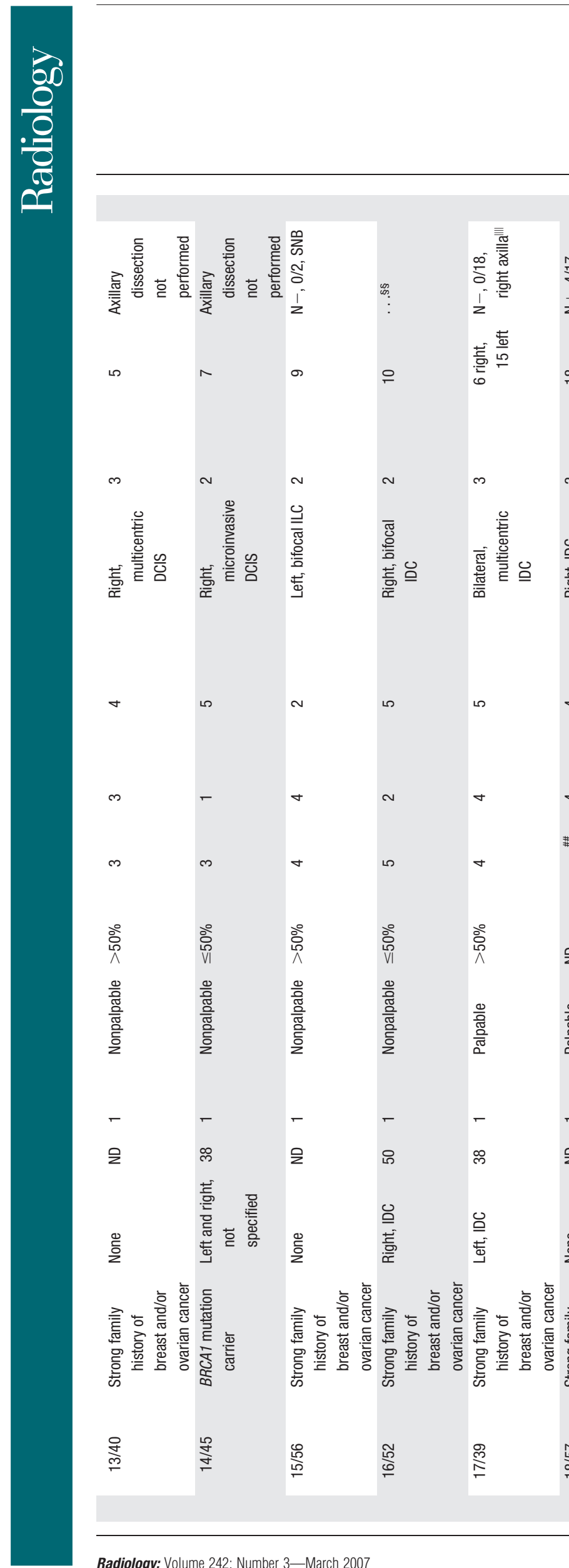

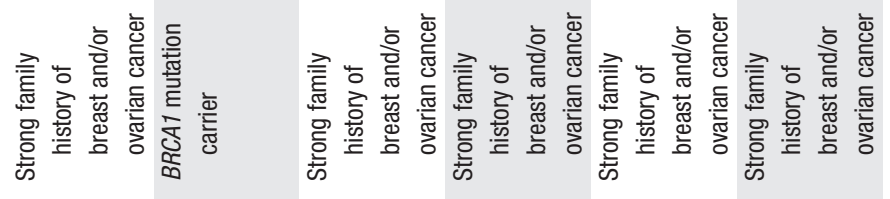

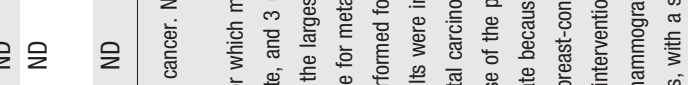

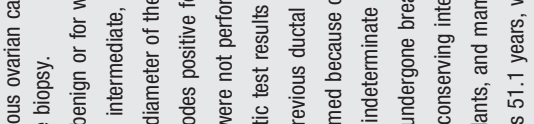

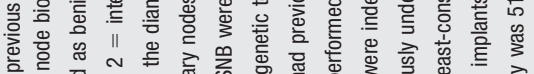
之 ₹

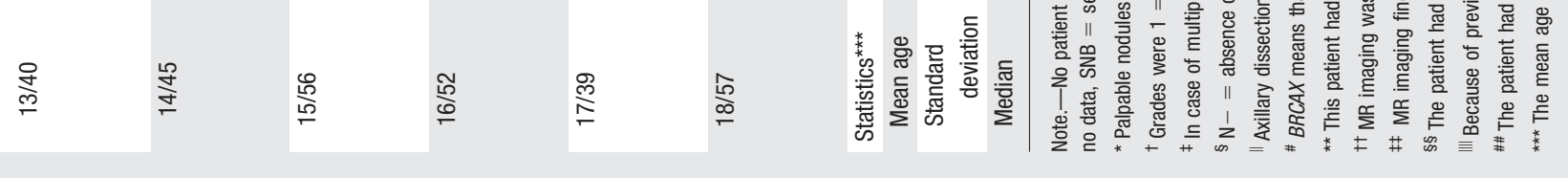


A difference with $P<.05$ was considered significant.

\section{Results}

\section{Screening Population}

The present interim analysis is based on the data available in March 2004 at the central data management unit in regard to the first round for the first 278 enrolled subjects and the second round for 99 subjects (ie, the first 99 subjects who underwent assessment in the second round), for a total of 377 screening events. The screening population (Table 2 ) consisted of 278 women (mean age, 46.0 years \pm 12.0 [standard deviation]; range, 25-79 years) enrolled by 17 centers. All 278 women underwent assessment in the first round and, until March 2004 , 99 women participated in the second round $(35.6 \%)$. One hundred seventy-five $(63 \%)$ of 278 women were enrolled because they were BRCA1 or BRCA2 mutation carriers or because they were first-degree relatives of BRCA1 or BRCA2 mutation carriers, whereas 103 (37\%) were enrolled on the basis of family history, according to the criteria stated before; seven of them, all with a previous breast and/or ovarian cancer, had an inconclusive genetic test (BRCAX status).

The mean ages of patients enrolled by using the two criteria stated previously were 45.8 years \pm 12.0 (175 women enrolled because they were BRCA1 or BRCA2 mutation carriers or first-degree relatives of BRCA1 or BRCA2 mutation carriers) and 46.5 years \pm 12.0 (103 women enrolled on the basis of the family history), and there was no significant difference (Mann-Whitney $U$ test) in mean age between them. In particular, the mean age of BRCA1 mutation carriers or first-degree relatives of BRCA1 mutation carriers was 45.7 years \pm 11.9 , whereas the mean age for the analogous BRCA2 group was 45.9 years \pm 12.3 , and there was no significant difference (MannWhitney $U$ test) in mean age between the groups. Of 278 women who underwent screening, 123 (44.2\%) had had previous breast and/or ovarian cancer and 155 (55.8\%) had not had these conditions; the mean ages at entry were 50.4 years \pm 11.5 for those who had had previous cancer and 42.6 years \pm 11.3 for those who had not had previous cancer; the difference between the groups was significant $(P<.001$, Mann-Whitney $U$ test $)$. The mean number of enrolled women per center was 16.3 (range, 4-62). The distribution among the centers was as follows: nine centers, four to 10 enrolled women; five centers, 11-30 enrolled women; and three centers, more than 30 enrolled women. For each of the 377 screening events, four examinations (CBE, mammography, US, and contrastenhanced MR imaging) were performed on the same day $(n=347)$, during 1 week $(n=1)$, during 2 weeks $(n=15)$, during 3 weeks $(n=2)$, during 4 weeks $(n=2)$, during 5 weeks $(n=1)$, during 6 weeks $(n=2)$, during 7 weeks $(n=2)$, and during 8 weeks $(n=5)$. All the MR examinations of fertile women were performed on the 7th-14th day of the menstrual cycle. A flow diagram of the entire study is presented in Figure 1.

\section{Cancer Detection}

Of 278 women who underwent screening at the first round, we found 11 patients with cancer (detection rate, $4.0 \%$ ), whereas of 99 women who underwent screening at the second round, we found seven patients with cancer (detection rate, $7.1 \%$ ), and the difference in rates was not significant $\left(\chi^{2}\right.$ test). The overall detection rate was $4.8 \%$ (18 of 377 ). The detection rates for enrollment criteria and presence or absence of personal history of breast or ovarian cancer are presented in Table 2.

The overall maximal pathologically determined mean diameter was 13.3 $\mathrm{mm} \pm 8.2$, and eight of 18 patients had tumors $10 \mathrm{~mm}$ or smaller in diameter. Fourteen patients had at least one invasive cancer and four patients had only in situ cancers; considering the largest tumor for each patient in both groups, the mean diameter was $15.8 \mathrm{~mm} \pm 7.6$ for the invasive cancers and $4.8 \mathrm{~mm} \pm 1.7$ for the in situ cancers. Nine (50\%) of 18 patients had multifocal, multicentric, or bilateral breast cancers. When we considered the most aggressive lesion in the nine patients with multifocal, multicentric, or bilateral cancers, we observed a percentage of patients with invasive cancers of $78 \%$ (14 of 18) (Table 3 ).

No interval cancer was reported. The sensitivity of contrast-enhanced MR imaging, mammography, and US is shown in Table 4. Mammography, which was not performed in one patient, aided in the diagnosis of a cancer in five $(63 \%)$ of eight patients with $50 \%$ or less of the breast occupied by fibroglandular density and in five (56\%) of nine patients with more than $50 \%$ of the breast occupied by fibroglandular density, and the difference was not significant (Fisher exact text); the only false-negative finding at contrast-enhanced MR imaging was in a breast with fibroglandular density that extended into more than $50 \%$ of the breast on the mammograms. Examples of a three-modality true-positive finding and of a finding that was true-positive only at contrast-enhanced MR imaging are shown in Figures 2 and 3. The false-negative finding at contrast-enhanced MR imaging is shown in Figure 4.

Of 18 patients in whom breast can-

\section{Table 4}

Patient-based Sensitivity and PPV of Contrast-enhanced MR Imaging, Mammography, US, and CBE in Multimodality Surveillance for Breast Cancer in 278 High-Risk Women

\begin{tabular}{llllll} 
& \multicolumn{2}{c}{ Sensitivity $(\%)$} & & \multicolumn{2}{c}{ PPV (\%) } \\
\cline { 2 - 3 } Modality & Value & $95 \% \mathrm{Cl}$ & & Value & $95 \% \mathrm{Cl}$ \\
\cline { 1 - 2 } Contrast-enhanced MR imaging & $93.8(15 / 16)$ & $71.7,98.9$ & & $62.5(15 / 24)$ & $42.7,78.8$ \\
Mammography & $58.8(10 / 17)$ & $36.0,78.4$ & & $76.9(10 / 13)$ & $49.7,91.8$ \\
US & $64.7(11 / 17)$ & $41.3,82.7$ & & $64.7(11 / 17)$ & $41.3,82.7$ \\
CBE & $50.0(9 / 18)$ & $29.0,71.0$ & & $81.8(9 / 11)$ & $52.3,94.9$
\end{tabular}

Note.-Numbers in parentheses were used to calculate the percentages. $\mathrm{Cl}=$ confidence interval. 


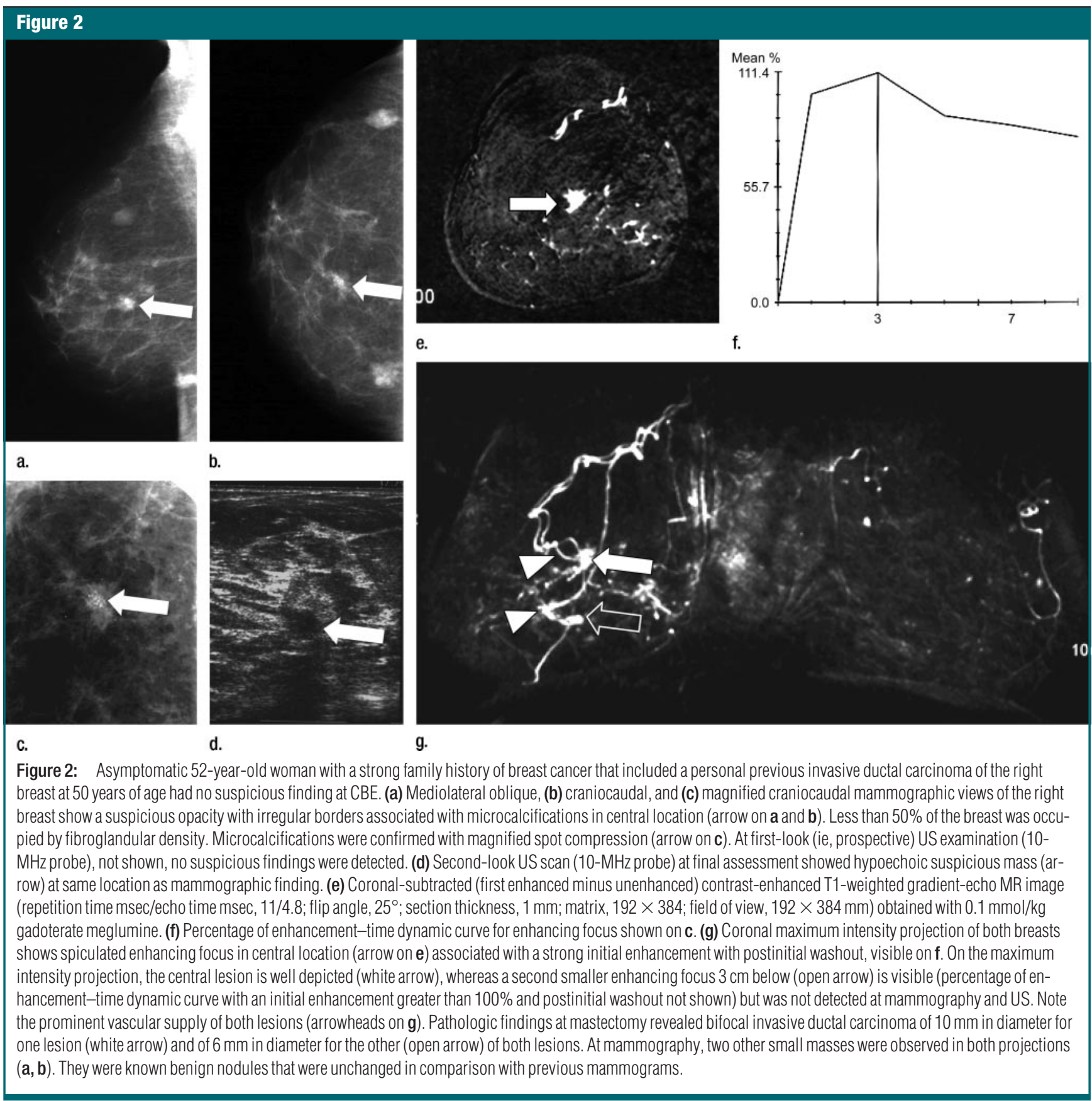

cer was detected, 11 had been enrolled on the basis of genetic testing and seven had been enrolled only on the basis of a strong family history of breast cancer and/or ovarian cancer. No significant difference was observed between the mean age of the seven BRCA1 mutation carriers with breast cancer (53.7 years \pm 8.3$)$ and that of the four BRCA2 mutation carriers (48.8 years \pm 16.3 )
(Mann-Whitney $U$ test), with a mean age of patients with BRCA1 and BRCA2 of 51.9 years \pm 11.3 (Table 5 ). In the 18 patients who presently had breast cancer, nine $(50 \%)$ had palpable tumors and nine $(50 \%)$ had nonpalpable tumors (Tables 6, 7).

In the 18 patients who had breast cancers, six $(33 \%)$ cancers were detected only with contrast-enhanced MR imaging. None of the cancers were detected only with CBE, only with mammography, or only with US (Table 8).

\section{Detection of Multiple Malignant Foci in Patients with Invasive Cancers}

When we considered the six patients with invasive cancers and multiple malignant foci per breast, we observed seven breasts with multiple 
foci. At CBE, the diagnosis of generically multiple palpable nodules was that they were suspicious for malignancy in one breast; the wrong diagnosis of unifocal cancer was determined in two breasts, and the diagnosis of all malignant lesions was missed in four. At mammography, underestimation of the number of malignant foci in one breast occurred, the wrong diagnosis of unifocal cancer was determined in four breasts, and the diagnosis of all malignant lesions was missed in two breasts. US, which was not performed in one patient with unilateral bifocal cancer, aided in the identification of the exact number of malignant foci in two breasts, contributed to underestimation of the number of malignant foci in one breast, and led to the wrong diagnosis of unifocal cancer in three breasts. At contrast-enhanced MR imaging, the number of malignant foci was correctly identified in four breasts and was underestimated in one breast, and the wrong diagnosis of unifocal cancer was determined in one breast. Moreover, contrast-enhanced MR imaging was the only technique that depicted a second focus of invasive lobular carcinoma, although it failed in the characterization of both foci as malignant (Table 3 , patient 15). Thus, the percentage of breasts with an exact detection of the number of malignant foci was $0 \%$ (zero of seven) for CBE and mammography, 33\% (two of six) for US, and 71\% (five of seven) for contrast-enhanced MR imaging.

\section{False-Positive Diagnoses and PPVs}

A total of 15 invasive procedures were performed in 13 patients without breast cancer who had a mean age of 43.9 years \pm 10.3 ; five of them were enrolled because they were BRCA1 mutation carriers, one was enrolled as a firstdegree relative of a BRCA1 mutation carrier, and seven were enrolled because of a strong family history of breast and/or ovarian cancer. Only two of them had a previous personal history of breast cancer. The following 20 falsepositive findings led to invasive diagnostic procedures: two at CBE, three at mammography, six at US, and nine at contrast-
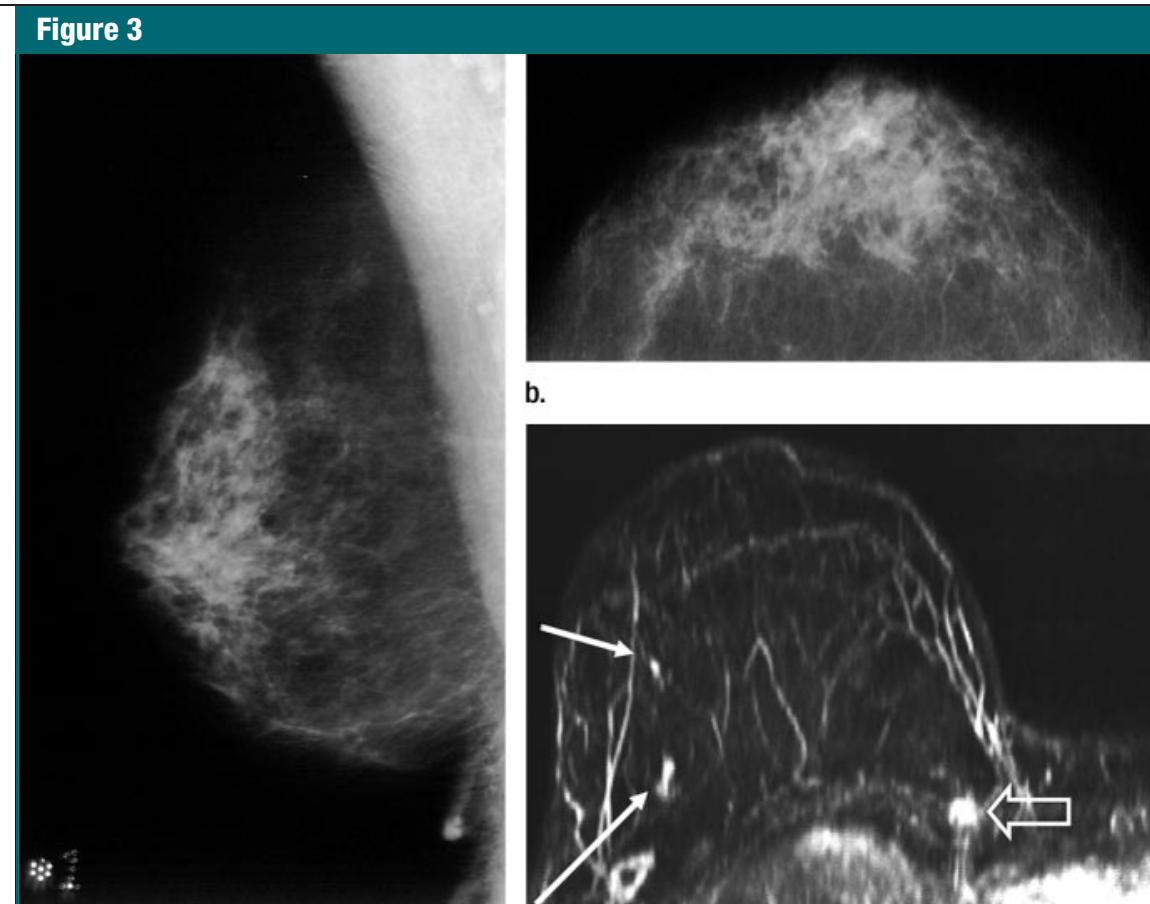

b.

a.

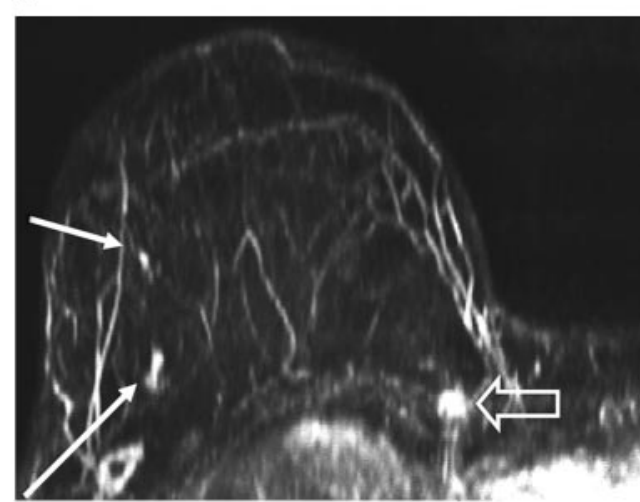

c.
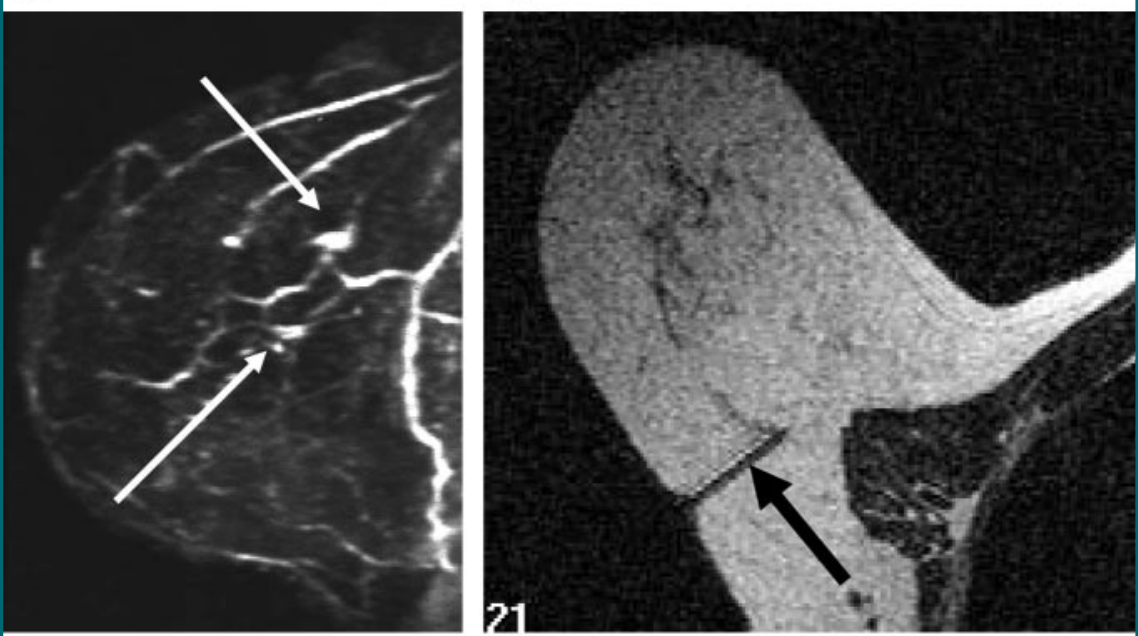

d.

e.

Figure 3: Asymptomatic 69-year-old BRCA1 carrier with previous left mastectomy for invasive ductal carcinomaat 55 years old had no suspicious finding at CBE of right breast. (a) Mediolateral and (b) craniocaudal mammographic views of right breast without any detected suspicious abnormality; less than $50 \%$ of breast was occupied by fibroglandular density. No suspicious finding was detected at US (not shown). (c) Transverse and (d) lateral maximum intensity projections of subtracted (first enhanced minus unenhanced) coronal T1-weighted gradient-echo contrast-enhanced MR images (8.9/ $4.3,25^{\circ}$ flip angle) after administration of $0.1 \mathrm{mmol} / \mathrm{kg}$ gadopentetate dimeglumine. Two small enhancing suspicious foci (solid arrows on cand d) are visible on the lateral quadrants of the right breast. (e) Transverse fast spin-echo MR imagingguided (204/20,90 flip angle) biopsy of one of the two foci confirmed presence of a malignant lesion. Arrow shows the amagnetic needle, with the tip at the site of the enhancing focus. Pathologic examination of the breastafter mastectomy demonstrated multiple foci of ductal carcinoma in situ (maximum diameter, $3 \mathrm{~mm}$ ); two were located where MR imaging showed enhancing lesions. In the inner posterior part of the breast, the transverse MR subtracted maximum intensity projection image shows enhancement caused by the superimposition of internal mammary vessels (open arrow on c). 


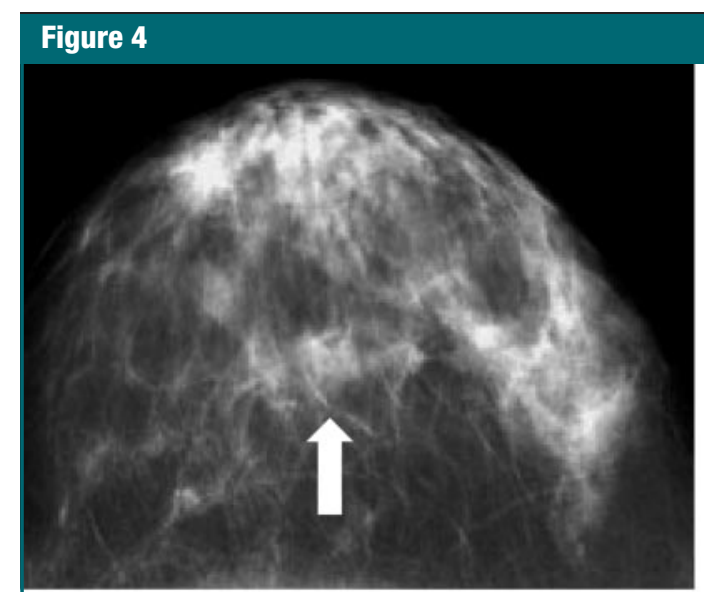

a.

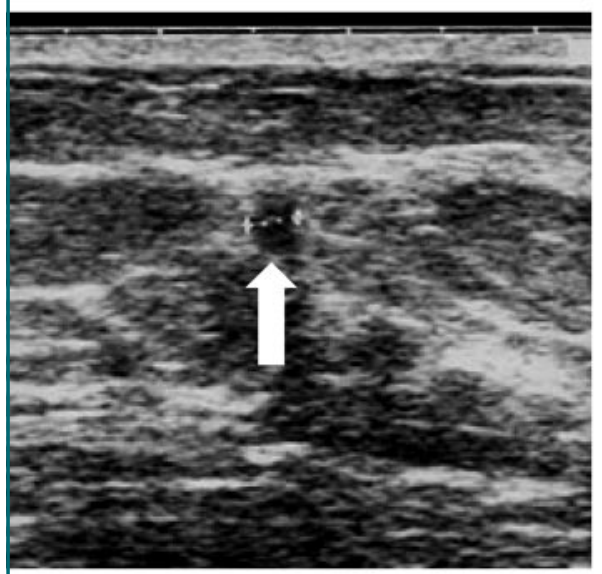

b.

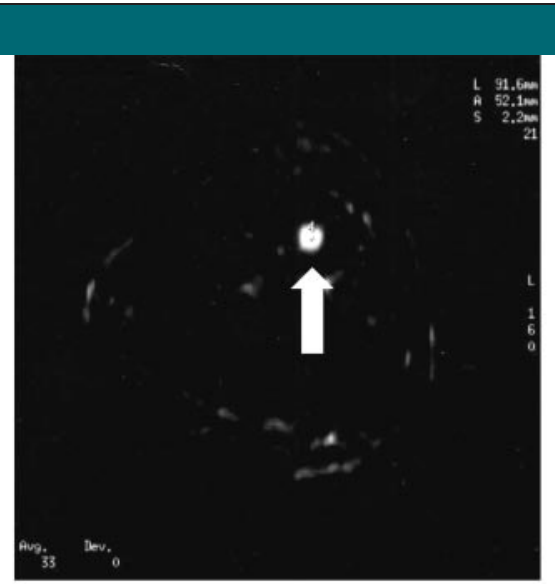

c.

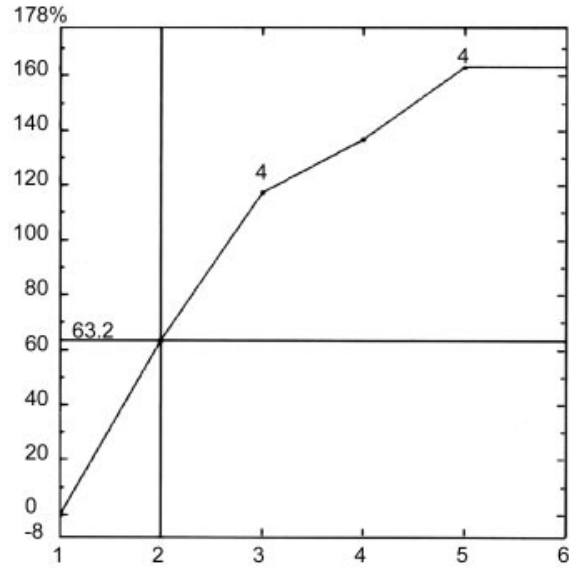

d.

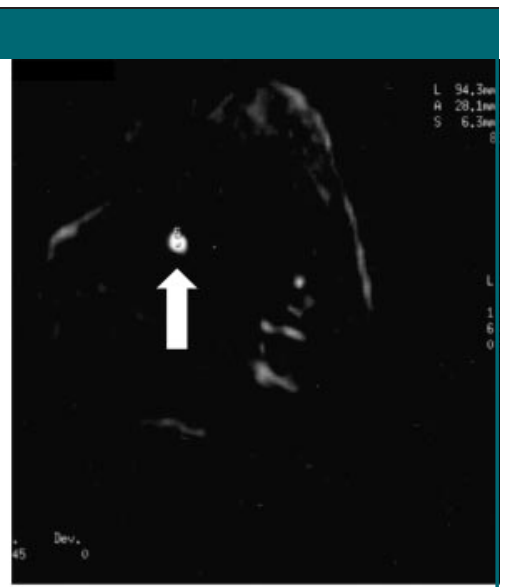

e.

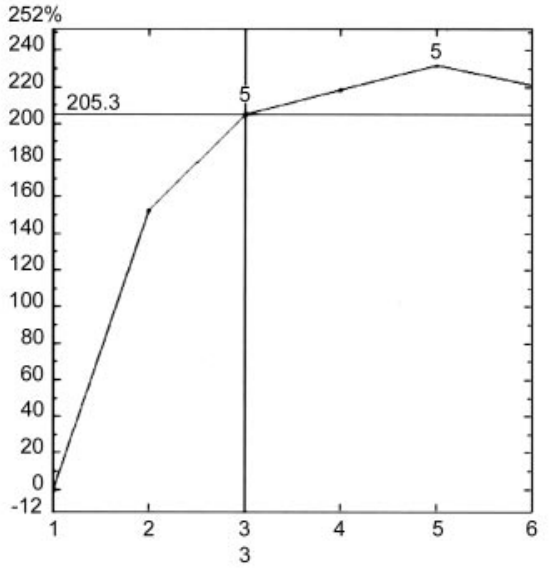

f.

Figure 4: Asymptomatic 56-year-old woman with a strong family history of breast cancer. (a) Craniocaudal mammographic view shows asymmetric opacity (arrow) in left breast (fibroglandular density greater than 50\%). (b) US scan shows hypoechoic mass (arrow) of $3 \mathrm{~mm}$ in diameter with partly irregular borders. Both mammogram and US scan were judged as suggestive of malignancy. (c, e) Two contrast-enhanced coronal subtracted (first enhanced minus unenhanced) T1-weighted gradient-echo MR images (8/4, $25^{\circ}$ flip angle, 2.5 -mm section thickness; $128 \times 256$ matrix; $175 \times 350$-mm field of view) obtained after administration of $0.1 \mathrm{mmol} / \mathrm{kg}$ gadopentetate dimeglumine. Each shows a round enhancing lesion (arrow). (d, f) Percentage of enhancement-time dynamic curves show continuous increase. Both enhancing lesions were judged as benign at MR imaging. Pathologic examination revealed two foci of invasive lobular carcinoma of 3 and $9 \mathrm{~mm}$ in diameter.

enhanced MR imaging. Because three invasive diagnostic procedures were determined with a concordant falsepositive finding at two modalities and one invasive diagnostic procedure was determined with a concordant falsepositive finding at three modalities, 20 false-positive findings led to only 15 invasive diagnostic procedures. These 15 invasive procedures were USguided fine-needle aspiration or coreneedle biopsy $(n=11)$, US-guided fine-needle aspiration and local excision $(n=2)$, fine-needle aspiration with mammographic stereotactic guidance $(n=1)$, and mastectomy $(n=1)$.
The woman who underwent mastectomy decided to undergo bilateral prophylactic mastectomy, which did not reveal any malignant lesions at pathologic examination; thus, one false-positive finding occurred at mammography. Of the 11 US-guided procedures, seven were performed as the result of second-look US findings detected only with contrast-enhanced MR imaging. The cytologic or pathologic benign diagnosis obtained with these 15 invasive procedures included one or two of the following findings: single or multiple fibroadenomas $(n=4)$, papilloma $(n=$ $2)$, adenosis or fibroadenosis $(n=2)$, intraparenchymal lymph node $(n=1)$, and fibrocystic changes or other benign findings $(n=8)$. On this basis, we calculated the PPVs (Table 4).

\section{Discussion}

The detection rate for breast cancer in high-risk women (in our study, 4.8\%) was more than 10 -fold that obtained in the screening of a general female population. The first-screening detection rate recently reported by Caines et al (43) by using mammography in the general female population was $0.37 \%$ for women aged $40-49$ years and $0.58 \%$ 
for women aged 50-59 years. We believe our high detection rate was caused by not only the high disease prevalence in the population but also the multimodality approach, which included contrast-enhanced MR imaging. In fact, in the trial by Brekelmans et al (44) in which patients underwent yearly screening mammography and CBE every 6 months, the detection rate in BRCA1 and BRCA2 mutation carriers was $3.3 \%$.

More important, we found no significant difference in detection rate in BRCA1 and BRCA2 mutation carriers or first-degree relatives of BRCA1 or BRCA2 mutation carriers compared with that in women enrolled only on the basis of a strong family history of breast cancer and/or ovarian cancer. The comparison between the cancers detected in the two groups of women enrolled according to these two selection criteria revealed no significant differences in terms of patients' ages, tumoral pathologic features, and axillary nodal involvement. This lack of a significant difference is due to the strict criteria we adopted for including women on the basis of only the family history and it also explains our high detection rate $(4.8 \%)$. This detection rate is identical to that obtained in the study by Warner et al (26), who included only BRCA1 and BRCA2 mutation carriers, with $39 \%$ of women with personal previous breast cancers; that percentage was $44 \%$ in our study.

In breast cancer screening programs for the general female population, women with a previous personal history of breast cancer are frequently excluded. This approach is under discussion (28) because no consensus exists about the duration and frequency of follow-up in women treated for breast cancer or about the schedule of examinations, particularly those at 5 years after the primary treatment (45). In our study, nine $(50 \%)$ of 18 patients who had a newly diagnosed breast cancer were women with a previous personal history of breast cancer, and the detection rate was $5.3 \%$, which was not significantly different from the detection rate $(4.3 \%)$ found in women without a previous personal breast cancer and/or ovarian cancer.

In women with familial-genetic predisposition to the disease, the risk of developing a contralateral breast cancer or an ovarian cancer within 5 years after a previous tumoral event is estimated to be between $30 \%$ and $60 \%$ $(4,46)$. Moreover, the inclusion of women with previous personal breast cancer and/or ovarian cancer is the probable reason for our relatively higher mean age at entry for the screen- ing population (46 years) when we compared that age with the age in two other studies in which women with a personal history of previous breast cancer were excluded $(25,27)$. In both of the studies, the mean or median age was 40 years. On the other hand, in the study by Warner et al (26), the percentage of women with a personal history of breast cancer was $39 \%$, which is close to our percentage of $44 \%$, and the reported mean age was 47 years.

Of the 11 patients genetically tested

\begin{tabular}{|c|c|c|}
\hline \multicolumn{3}{|c|}{$\begin{array}{l}\text { Comparison between } 11 \text { BRCA1 and BRCA2 Mutation Carriers and Seven Women } \\
\text { Enrolled on the Basis of a Strong Family History of Breast Cancer and/or Ovarian } \\
\text { Cancer in } 278 \text { High-Risk Women }\end{array}$} \\
\hline Clinical Data & Group A & Group B \\
\hline Patient age $(y)$ & $51.9 \pm 11.3$ & $49.9 \pm 8.8$ \\
\hline Diameter at pathologic examination (mm) & $14.1 \pm 9.4$ & $12.1 \pm 6.4$ \\
\hline Invasive breast cancers* & $9(82)$ & $5(71)$ \\
\hline Pathologic grade of cell differentiation & $2.4 \pm 0.8$ & $2.5 \pm 0.7$ \\
\hline Multifocal, multicentric, or bilateral cancers ${ }^{\star}$ & $4(37)$ & $5(71)$ \\
\hline Axillary nodal involvement ${ }^{\star}$ & $1(9)$ & $2(29)$ \\
\hline
\end{tabular}

Note.-Group A included 11 women who were BRCA1 and BRCA2 mutation carriers who had cancer, and group B included seven women with a strong family history of breast cancer and/or ovarian cancer who had cancer. Data are the mean \pm standard deviation except where otherwise specified. The Fisher exact test was used for comparisons between percentages, and the Mann-Whitney $U$ test was used for comparisons between patients' ages, between tumoral diameters at pathologic examinations, and between pathologic grades of cell differentiation. Differences between group A and group B were not significant.

* Data are numbers of women, and numbers in parentheses are percentages.

\section{Table 6}

Comparison between Nine Patients with Palpable Cancers and Nine Patients with Nonpalpable Cancers Detected in Multimodality Screening in 278 Women at Genetic-Familial High Risk for Breast Cancer

\begin{tabular}{llc} 
Clinical Data & Group A & Group B \\
\hline Patient age (y) & $47.2 \pm 9.1$ & $55.0 \pm 10.2$ \\
Diameter at pathologic examination (mm) & $19.0 \pm 6.9$ & $7.7 \pm 4.8$ \\
Invasive breast cancers & $9 / 9(100)$ & $6 / 9(67)$ \\
Pathologic grade of cell differentiation $^{\star}$ & $2.7 \pm 0.7$ & $2.1 \pm 0.8$ \\
Multifocal, multicentric, or bilateral cancers & $3 / 9(33)$ & $6 / 9(67)$ \\
Axillary nodal involvement* $^{\star}$ & $2 / 8(25)$ & $1 / 10(10)$
\end{tabular}

Note.-Group A included nine patients with palpable cancers, and group B included nine patients with nonpalpable cancers. Data are the mean \pm standard deviation except where otherwise specified. Of the nine patients with palpable cancers, one with marked anxiety did not undergo MR imaging, one had indeterminate results of MR imaging because of movement artifacts, and one with breast implants did not undergo successful mammography because of marked discomfort during compression. Of the nine patients with nonpalpable cancers, one did not undergo US for unspecified reasons. The Fisher exact test was used for comparisons between percentages, and the Mann-Whitney $U$ test was used for comparisons between patients' ages, between tumoral diameters at pathologic examination, and between pathologic grades of cell differentiation. Differences between group A and group B were not significant except for diameter at pathologic examination $(P<.002)$.

${ }^{*}$ Data are numbers of women, and numbers in parentheses are percentages. 


\section{Table 7}

Patient-based Sensitivity of Contrast-enhanced MR Imaging, Mammography, and US for Palpable and Nonpalpable Breast Cancers in Multimodality Screening in 278 Women at Genetic-Familial High Risk for Breast Cancer

\begin{tabular}{lcl} 
Modality & $\begin{array}{l}\text { Sensitivity for Palpable } \\
\text { Cancers (\%) }\end{array}$ & $\begin{array}{l}\text { Sensitivity for Nonpalpable } \\
\text { Cancers }(\%)\end{array}$ \\
\hline Contrast-enhanced MR imaging & $100.0(7 / 7)$ & $88.9(8 / 9)$ \\
Mammography & $87.5(7 / 8)$ & $33.3(3 / 9)$ \\
US & $100.0(9 / 9)$ & $25.0(2 / 8)$ \\
\hline
\end{tabular}

Note.-Numbers in parentheses were used to calculate the percentages.

Table 8

Comparison between 12 Patients with Tumors Detected with a Combination of CBE, Mammography, and/or US and Six Patients with Tumors Detected Only with Contrast-enhanced MR Imaging in 278 High-Risk Women

Clinical Data

Patient age (y)

Diameter at pathologic examination $(\mathrm{mm})$

Invasive cancers*

Pathologic grade of cell differentiation

Multifocal, multicentric, or bilateral cancers

Axillary nodal involvement*

Group A

Group B

$48.6 \pm 8.2$

$18.0 \pm 7.3$

11 (92)

\section{$2.5 \pm 0.7$}

$6(50)$

$2(17)$
$56.2 \pm 12.5$

$5.2 \pm 1.5$

$3(50)$

$2.0 \pm 1.0$

$3(50)$

1 (17)

Note.-Group A included 12 patients with tumors detected with a combination of CBE, mammography, and US, and group B included six patients with tumors detected only with contrast-enhanced MR imaging. For group A, of the 12 tumors, 11 were true-positive and one was false-negative at MR imaging. Data are the mean \pm standard deviation except where otherwise specified. The Fisher exact test was used for comparisons between percentages, and the Mann-Whitney $U$ test was used for comparisons between ages, between tumoral diameters at pathologic examination, and between pathologic grades of cell differentiation. The differences between group $A$ and group $B$ were not significant except for diameter at pathologic examination $(P<.001)$. For invasive cancers, $P=.078$.

* Data are numbers of patients, and numbers in parentheses are percentages.

in whom breast cancer was detected, seven were BRCA1 mutation carriers and four were BRCA2 mutation carriers. The relatively advanced mean age of the BRCA1 and BRCA2 mutation carriers (53.7 years \pm 8.3 and 48.8 years \pm 16.3 , respectively) and the higher age (even though not significantly different) of the BRCA1 group can be explained by the presence of patients with a personal history of previous breast cancer in both groups: five $(71 \%)$ of seven in the BRCA1 mutation carrier group and one (25\%) of four in the BRCA2 mutation carrier group.

The mean tumoral diameter at pathologic examination was $13.7 \mathrm{~mm} \pm$ 8.6, and eight (44\%) of 18 patients had tumors of $10 \mathrm{~mm}$ or smaller in diameter; the percentage of patients with tumors of that diameter was similar to the percentage reported by other authors, and the percentages ranged from $43 \%$ to $55 \%(25-27)$. This result appears to be interesting if we consider that the tumor volume doubling time of invasive cancers is shorter in BRCA1 or BRCA2 mutation carriers than it is in noncarriers, as recently reported (ie, 45 days for carriers and 84 days for noncarriers) (47).

The high percentage (78\%) of invasive cancers in our study is in agreement with data in previous studies; in those studies, the researchers reported a percentage from $73 \%$ (26) to $88 \%$ (25). Our percentage of cancers with a pathologic grade of cell differentiation of $3(10$ of $18,56 \%)$ is slightly lower than that reported in two previous studies about high-risk women; in those studies, this percentage was $66 \%$ (27) and $67 \%$ (24). Interestingly, Kriege et al (25), who included a large proportion of women with a lower risk of breast cancer (starting from only a $15 \%$ of lifetime risk) in their study, reported a percentage of grade 3 cancers of only $37 \%$.

On the other hand, of 13 patients with invasive cancers in whom the axillary nodal status was explored, we had only three patients with nodal cancer involvement (23\%), confirming the possibility of an early diagnosis in terms of nodal involvement in high-risk women, as already found in other studies in which the percentages of invasive cancers with known nodal status and metastatic involvement ranged from 0\% (24) to $21 \%$ (25). For comparison, we should consider that several screening studies of high-risk women by using mammography without contrast-enhanced MR imaging have shown an incidence of positive nodes of $30 \%-45 \%(44,48,49)$.

The absence of interval cancer in our series must be related to the small number of screening events per woman (only 1.4) considered in this report and to a limited follow-up. The higher detection rate at the second round, which was $7.1 \%$ and was greater but not significantly higher than $4.0 \%$ at the first round, confirms that in high-risk women we should adjust some conceptual tools used in mammographic screening of the general female population. For instance, the typical difference between "prevalent" cancers of the first screening and "incident" cancers of subsequent screenings was not observed in our study. Similar results were reported by Warner et al (26), who described a detection rate of $5.5 \%$ at the first round and $5.1 \%$ at the second round. This finding is probably related to the high speed of tumoral growth of breast cancer in high-risk women $(26,47)$.

Our study findings indicate that contrast-enhanced MR imaging may enable the detection of unsuspected breast cancers by using other diagnostic modalities in women at genetic-familial high risk, as already reported in other studies (23-27). In our study, the high sensitivity of $94 \%$ for contrast-enhanced MR imaging could be related to the lower number of screening events 
per woman, which was 1.4 without interval cancers, when we compared these values with those in the study by Kriege et al (sensitivity, 71\%; screening events per woman, 2.7; interval cancers, four) (25). In both the study by Warner et al (26) and the Magnetic Resonance Imaging in Breast Screening study by Leach et al (27), the researchers reported a sensitivity of $77 \%$ for contrast-enhanced MR imaging. Warner et al reported 1.9 screening events per woman with only one interval cancer, and Leach et al reported 3.0 screening events per woman with two interval cancers. Kuhl et al (24), however, reported a sensitivity of $100 \%$ and 1.9 screening events per woman without interval cancers. On the other hand, if the indeterminate MR result caused by artifacts from the patient's movements is considered false-negative, we obtained a sensitivity of $88 \%$ (15 of 17). In this patient, however, contrast-enhanced MR imaging was not repeated because we had a concordant positive result with CBE, mammography, and US.

We did not observe a higher prevalence of palpable cancers at the first round: The percentage of palpable cancers was $36 \%$ (four of 11 ) at the first round and $57 \%$ (four of seven) at the second round. Palpability was clearly related to the mean tumoral diameter $(19.0 \mathrm{~mm} \pm 6.9$ for nine palpable cancers vs $7.7 \mathrm{~mm} \pm 4.8$ for nine nonpalpable cancers), and this high percentage of palpable cancers could be related to the rapid tumoral growth in these patients (47). We confirm the insufficient sensitivity of CBE in high-risk women, as already reported (24-26).

The range of sensitivity values (88\%-100\%) for palpable cancers among the three imaging modalities in our study compared with that for nonpalpable cancers (33\% for mammography, $25 \%$ for US, and $89 \%$ for contrast-enhanced MR imaging) shows that the advantage of contrast-enhanced MR imaging is in the detection of small nonpalpable cancers. It should be emphasized that the mean age of patients in whom cancers were detected with only contrast-enhanced MR imaging was 56.2 years \pm 12.5 , and this age is relatively more advanced, even though it is not significantly higher than the mean age of 48.6 years \pm 8.2 in women in whom diagnosis was determined with a combination of CBE, mammography, and US. In other words, we do not have evidence to advocate a gain in sensitivity by using contrast-enhanced MR imaging only in younger premenopausal highrisk women and not in older postmenopausal high-risk women. Also, the breast density pattern does not seem to play a key role, as shown by the similar low sensitivity for mammography in both breasts with fibroglandular density of $50 \%$ or less (five of eight, 63\%) and breasts with fibroglandular density greater than $50 \%$ (five of nine, $56 \%$ ). Moreover, the possible overdiagnosis because of the high sensitivity of contrast-enhanced MR imaging is counterbalanced by the fact that five of six patients in whom breast cancer was diagnosed during the study only with contrast-enhanced MR imaging had an invasive or multifocal, multicentric, or bilateral cancer.

The PPV of $63 \%$ for contrast-enhanced MR imaging in our experience is very close to that of $64 \%$ reported by Kuhl et al (24) and higher than that of $46 \%$ reported by Warner et al (26). Our performance can be related to the use of a model of interpretation of contrastenhanced MR images in which both morphologic and dynamic parameters are integrated in a simple score $(38,39)$, which can be easily translated into one of the BI-RADS categories of 1-5. The key point is that, with this scoring system, a round, oval, or lobular homogeneously enhancing lesion with regular margins, less than $100 \%$ initial enhancement, and a continuous or plateau postinitial enhancement (or with an initial enhancement of $100 \%$ or greater and a continuous postinitial enhancement) is considered benign. We paid a trade-off for this criterion: The only false-negative finding at contrast-enhanced MR imaging was an invasive lobular carcinoma that was diagnosed as benign. Sensitivity, however, remained as high as $94 \%$, and a good balance with PPV (63\%) was obtained. Nevertheless, it is important to realize that our results (in particular the PPV of $63 \%$ for contrast-enhanced MR imaging in a screening setting) were obtained in a very high-risk population and that the same protocol in a lower-risk population could have a lower performance.

Our PPV for mammography (77\%) is within the range of values in similar studies: from $30 \%(24)$ to $89 \%$ (26). We observed a higher PPV for US (65\%) if one compares the PPV with that of $29 \%$ from one previous study (26) and also a higher PPV for CBE $(82 \%)$ if one compares the PPV with those of $18 \%$ (25) and 50\% (26) from two previous studies.

The first general limitation of our study was the nonrandomized study design. We do not know whether an intensive screening protocol (including contrast-enhanced MR imaging) is able to provide an effect in terms of saved years of life in such high-risk women. Such a question could be answered with randomized trials, although we agree with the ethical concerns recently raised about this possibility (50).

The second important limitation was the small number of screening events per woman considered in the study (99 women for the first and second rounds and 179 women for only the first round, for a total of 377 screening events and a mean of 1.4 screening events per woman). We should consider, however, that very few interval cancers were reported in prospective studies in which contrast-enhanced MR imaging was used for the screening of high-risk women: a total of seven interval cancers versus 104 cancers detected during the trials for 2956 women enrolled and 2.7 screening events per woman (24-27).

The third limitation was that paired statistical analysis of the diagnostic performance of the modalities was not performed. This comparison will be performed at the end of the trial.

In conclusion, our interim experience suggests that contrast-enhanced MR imaging is highly sensitive (94\%) for detection of breast cancer in multimodality yearly surveillance-which also includes CBE, mammography, and US- 
in high-risk women; moreover, the PPV of contrast-enhanced MR imaging (63\%) is close to that of US (65\%). In addition, this multimodality approach allows an early cancer diagnosis in terms of tumoral size and nodal involvement. Furthermore, our data suggest that addition of contrast-enhanced MR imaging to the screening regimen may enable detection of otherwise unsuspected breast cancer not only in women who are BRCA1 and BRCA2 mutation carriers but also in women who meet strict criteria for a strong family history of breast cancer and/or ovarian cancer and that, in both groups, women with a personal history of breast cancer should be included. Finally, our findings support the high sensitivity of contrast-enhanced MR imaging of the breast in the detection of multiple malignant foci in high-risk women.

Acknowledgments: F.P. was responsible for the project. F.S. operated radiological central coordination. Data management was operated in cooperation with A.V. and M.S.

Enrolling centers: The enrolling centers, cities in which they are located, and other persons who cooperated in this study are as follows: Istituto Nazionale per lo Studio e la Cura dei Tumori, Milan: Silvana Bergonzi, Claudia Costa, Claudio Ferranti, Monica Marchesini, Gianfranco Scaperotta, Laura Suman, Daniele Vergnaghi; Centro di Riferimento Oncologico, Aviano: Riccardo Dolcetti, Alessandra Viel, Silvia Venturini; University of Modena and Reggio Emilia, Modena: Renato Romagnoli, Rachele Battista, Barbara Canossi, Mario de Santis, Isabella Marchi, Veronica Medici, Daniela Turchetti; University Hospital of Ferrara, Ferrara: Patrizia Querzoli; Ordine Mauriziano, Istituto per la Ricerca e la Cura del Cancro, Candiolo, Turin: Lisa Cellini, Laura Martincich, Daniele Regge; University of Pisa, Pisa: Carlo Bartolozzi, Generoso Bevilacqua, Isa Brunetti, Maria Adelaide Caligo, Claudia Giaconi, Chiara Iacconi, Dionisa Mazzotta, Monica Moretti, Manuela Roncella; University of Padua, Padua: Grazia Artioli, Emma D’Andrea, Gianni Nardelli, Maria Ornella Nicoletto, Luigi Pescarini, Giorgio Zavagno; Policlinico San Donato, San
Donato Milanese, Milan: Bijan Babaei, Francesco Gerra, Adamo Magaldi, Andrea Iozzelli, Eleonora N. Lupo, Michela Russo; European Institute of Oncology, Milan: Monica Barile, Luca Bazzi, Enrico Cassano, Andrea Decensi, Massimo Bellomi, Bernardo Bonanni, Irene Feroce, Gaetano Villa; University of Udine, Udine: Massimo Bazzocchi, Alexia Bestagno, Giuliana Francescutti; Federico II University, Naples: Matilde Pensabene, Ida Capuano; San Martino University Hospital, Genoa: Davide Brizzi; University of Chieti, Chieti: Pasquale Battista, Alessandro Cama, Alessandro Carriero, Renato Mariani-Costantini, Giandomenico Palka; University of L'Aquila, L'Aquila: Antonietta Ciccozzi, Franco Colista, Caterina Fiumara, Silvia Lelli, Maria Mancini, Carlo Masciocchi, Enrico Ricevuto; University of Trieste, Trieste: Antonio Amoroso, Carla Dellach, Giorgio Mustacchi, Roberto Pozzi-Mucelli; and VitaSalute University, San Raffaele Hospital, Milan: Angela De Gaspari, Isabella Fedele.

Authors: The list of remaining authors follows: Anna Cilotti, MD, Cosimo Di Maggio, MD, Alfonso Fausto, MD, Lorenzo Preda, MD, Chiara Zuiani, MD, Alma Contegiacomo, MD, Antonio Orlacchio, MD, Massimo Calabrese, MD, Lorenzo Bonomo, MD, Ernesto Di Cesare, MD, Maura Tonutti, MD, Pietro Panizza, MD, and Alessandro Del Maschio, MD.

Author affiliations: Unit of Radiology, IRCCS Policlinico San Donato, San Donato Milanese, Milan, Italy (A.F.); Department of Cell Biology and Neurosciences (G.D.) and National Center of Epidemiology, Surveillance, and Health Promotion (A.V., M.S.), Istituto Superiore di Sanità, Rome, Italy; Departments of Diagnostic Radiology 1 (R.M., G.T.) and Medical Genetics Service, Experimental Oncology (S. Manoukian), Istituto Nazionale per lo Studio e la Cura dei Tumori, Milan, Italy; Departments of Radiology (S. Morassut) and Medical Oncology (C.d.G.), Centro di Riferimento Oncologico, Aviano, Italy; Department of Oncology and Hematology, University of Modena and Reggio Emilia, Modena, Italy (M.F., L.C.); Breast Imaging Unit, University Hospital of Fer- rara, Ferrara, Italy (S. Corcione); Department of Diagnostic Radiology, Ordine Mauriziano, Istituto per la Ricerca e la Cura del Cancro, Candiolo, Turin, Italy (S. Cirillo, V.M.); Division of Diagnostic and Interventional Radiology, Department of Oncology, Transplants, and Advanced Technologies in Medicine, University of Pisa, Pisa, Italy (A. Cilotti); Section of Senology and Diagnostic Imaging, Department of Oncology and Surgical Sciences, University of Padua, Padua, Italy (C.D.M.); Department of Radiology, European Institute of Oncology, Milan, Italy (L.P.); Institute of Radiology, University of Udine, Udine, Italy (C.Z.); Department of Endocrinology and Molecular and Clinical Oncology, Federico II University, Naples, Italy (A. Contegiacomo); Department of Radiology and Diagnostic Imaging, General Hospital San Giovanni Calibita Fatebene Fratelli, Rome, Italy (A.O., now at the Department of Diagnostic Imaging and Interventional Radiology, Tor Vergata University, Rome, Italy); Department of Radiology, University of Genoa, Genoa, Italy (M.C.); Department of Clinical Sciences and Bioimages, University of Chieti, Chieti, Italy (L.B., now at the Department of Radiology, Catholic University, Rome, Italy); Department of Radiology, University of L'Aquila, L'Aquila, Italy (E.D.C.); Department of Radiology, University of Trieste, Trieste, Italy (M.T.); and Department of Radiology, Vita-Salute University, San Raffaele Hospital, Milan, Italy (P.P., A.D.M.).

Author contributions: Guarantors of integrity of entire study, F.S., F.P.; study concepts/study design or data acquisition or data analysis/interpretation, all authors; manuscript drafting or manuscript revision for important intellectual content, all authors; manuscript final version approval, all authors; literature research, F.S., F.P., G.D., S. Manoukian, A.F.; clinical studies, R.M., G.T., S. Morassut, C.d.G., S. Corcione, S. Cirillo, V.M., A. Cilotti, C.D.M., A.F., L.P., C.Z., A. Contegiacomo, A.O., M.C., E.D.C., M.T., P.P., A.D.M.; statistical analysis, F.S., A.V., M.S., A.F.; and manuscript editing, F.S., F.P., S. Manoukian 


\section{References}

1. Jemal A, Tiwari RC, Murray T, et al. Cancer statistics, 2004. CA Cancer J Clin 2004;54: $8-29$.

2. Siesling S, van Dijck JA, Visser O, Coebergh JW; Working Group of the Netherlands Cancer Registry. Trends in incidence of and mortality from cancer in the Netherlands in the period 1989-1998. Eur J Cancer 2003; 39:2521-2530

3. Israeli D, Tartter PI, Brower ST, Mizrachy B, Bratton J. The significance of family history for patients with carcinoma of the breast. J Am Coll Surg 1994;179:29-32.

4. Hoskins KF, Stopfer JE, Calzone KA, et al. Assessment and counseling for women with a family history of breast cancer: a guide for clinicians. JAMA 1995;273:577-585.

5. Claus EB, Schildkraut JM, Thompson WD, Risch NJ. The genetic attributable risk of breast and ovarian cancer. Cancer 1996;77: $2318-2324$.

6. Ford D, Easton DF, Stratton M, et al. Genetic heterogeneity and penetrance analysis of the BRCA1 and BRCA2 genes in breast cancer families. Am J Hum Genet 1998;62: $676-689$.

7. Podo F, Sardanelli F, Canese R, et al. The Italian multi-centre project on evaluation of MRI and other imaging modalities in early detection of breast cancer in subjects at high genetic risk. J Exp Clin Cancer Res 2002; 21 (suppl 3):115-124.

8. Serova OM, Mazoyer S, Puget N, et al. Mutations in BRCA1 and BRCA2 in breast cancer families: are there more breast cancer susceptibility genes? Am J Hum Genet 1997; 60:486- 495 .

9. Szabo CI, King MC. Population genetics of BRCA 1 and BRCA2. Am J Hum Genet 1997; 60:1013-1020.

10. Krainer M, Silva Arrieta S, Fitzgerald MG, et al. Differential contribution of BRCA1 and BRCA2 to early-onset breast cancers. N Engl J Med 1997;336:1416-1421.

11. Pathology of familial breast cancer: differences between breast cancers in carriers of BRCA1 or BRCA2 mutations and sporadic cases. Breast Cancer Linkage Consortium. Lancet 1997;349:1505-1510.

12. Lakhani SR, Jacquemier J, Sloane JP, et al. Multifactorial analysis of differences between sporadic breast cancers and cancers involving BRCA1 and BRCA2 mutations. J Natl Cancer Inst 1998;90:1138-1135.

13. Porter DE, Dixon M, Smyth E, Steel CM. Breast cancer survival in BRCA1 carriers. Lancet 1993;341:184-185.
14. Verhoog LC, Brekelmans CT, Seynaeve C, et al. Survival and tumour characteristics of breast-cancer patients with germline mutations of BRCA1. Lancet 1998;351:316-321.

15. Lehman CD, Schnall MD. Imaging in breast cancer: magnetic resonance imaging. Breast Cancer Res 2005;7:215-219.

16. Heywang-Kobrunner SH, Heinig A, Pickuth $\mathrm{D}$, et al. Interventional MRI of the breast: lesion localization and biopsy. Eur Radiol 2000; $10: 36-45$.

17. Liberman L, Morris EA, Dershaw DD, et al. Fast MRI-guided vacuum-assisted breast biopsy: initial experience. AJR Am J Roentgenol 2003;181:1283-1293.

18. Kuhl CK, Kreft BP, Bieling HB, et al. Dynamic breast MRI in premenopausal healthy volunteers: normal values of contrast enhancement and cycle phase dependency. Radiology 1997;203:137-144

19. Müller-Schimpfle M, Ohmeauser K, Stoll P, Dietz K, Claussen CD. Menstrual cycle and age: influence on parenchymal contrast medium enhancement in MR imaging of the breast. Radiology 1997;203:145-149.

20. Liberman L, Morris EA, Benton CL, Abramson AF, Dershaw DD. Probably benign lesions at breast magnetic resonance imaging: preliminary experience in high-risk women. Cancer 2003;98:377-388.

21. Stoutjesdijk MJ, Boetes C, Jager GJ, et al. Magnetic resonance imaging and mammography in women with a hereditary risk of breast cancer. J Natl Cancer Inst 2001;93: 1095-1102.

22. Morris EA, Liberman L, Ballo DJ. MRI of occult breast carcinoma in a high-risk population. AJR Am J Roentgenol 2003;181:619 626.

23. Tilanus-Linthorst MM, Obdeijn IM, Bartels KC, de Koning HJ, Oudkerk M. First experiences in screening women at high risk for breast cancer with MR imaging. Breast Cancer Res Treat 2000;63:53-60.

24. Kuhl CK, Schmutzler RK, Leutner CC, et al. Breast MR imaging screening in 192 women proved or suspected to be carriers of a breast cancer susceptibility gene: preliminary results. Radiology 2000;215:267-279.

25. Kriege M, Brekelmans CT, Boetes C, et al. Efficacy of MRI and mammography for breast cancer screening in women with a familial or genetic predisposition. N Engl J Med 2004;351:427-437.

26. Warner E, Plewes DB, Hill KA, et al. Surveillance of BRCA1 and BRCA2 mutation carriers with magnetic resonance imaging, ultrasound, mammography, and clinical breast examination. JAMA 2004;292:1317-1325.
27. Leach MO, Boggis CR, Dixon AK, et al. Screening with magnetic resonance imaging and mammography of a UK population at high familial risk of breast cancer: a prospective multicentre cohort study (MARIBS). Lancet 2005;365:1769-1778.

28. Sardanelli F, Podo F. Women with history of breast cancer excluded from screening programs: is it the right choice? [letter]. Radiology $2005 ; 234: 971$.

29. Grompe M. The rapid detection of unknown mutations in nucleic acids. Nat Genet 1993; 5:111-117.

30. Rosenblum BB, Lee LG, Spurgeon SL, et al. New dye-labeled terminators for improved DNA sequencing patterns. Nucleic Acids Res 1997;25:4500 - 4504 .

31. Yager TD, Baron L, Batra R, et al. High performance DNA sequencing, and the detection of mutations and polymorphisms, on the Clipper sequencer. Electrophoresis 1999;20:1280-1300.

32. Kourkine IV, Hestekin CN, Barron AE Technical challenges in applying capillary electrophoresis-single strand conformation polymorphism for genetic analysis. Electrophoresis 2002;23:1375-1385.

33. Jones AC, Austin J, Hansen N, et al. Optimal temperature selection for mutation detection by denaturing HPLC and comparison to single-stranded conformation polymorphism and heteroduplex analysis. Clin Chem 1999; 45:1133-1140.

34. American College of Radiology. Reporting system. In: Breast imaging reporting and data system (BI-RADS). 3rd ed. Reston, Va: American College of Radiology, 1998.

35. Mendelson EB, Berg WA, Merrit CR. Toward a standardized breast ultrasound lexicon: BIRADS-ultrasound. Semin Roentgenol 2001; 36:217-225.

36. Sardanelli F, Iozzelli A, Fausto A. MR imaging of the breast: indications, established technique and new directions. Eur Radiol 2003;13(suppl 3):N28-N36.

37. Sardanelli F, Fausto A, Iozzelli A, Rescinito G, Calabrese M. Dynamic breast magnetic resonance imaging: effect of changing the region of interest on early enhancement using 2D and 3D techniques. J Comput Assist Tomogr 2004;28:642-646.

38. Fischer U, Kopka L, Grabbe E. Breast carcinoma: effect of preoperative contrastenhanced MR imaging on the therapeutic approach. Radiology 1999;213:881-888.

39. Baum F, Fischer U, Vosshenrich R, Grabbe E. Classification of hypervascularized lesions in CE MR imaging of the breast. Eur Radiol 2002;12:1087-1092. 
40. Liberman L, Menell JH. Breast imaging reporting and data System (BI-RADS). Radiol Clin North Am 2002;40:409-430.

41. Cancer statistics in developing countries: report on a WHO meeting, Nagoya, Japan, 18-22 August, 1981. World Health Stat Q 1983;36:213-217.

42. Glantz SA. Primer of biostatistics. 4th ed. New York, NY: McGraw-Hill, 1997; 140-150.

43. Caines JS, Schaller GH, Iles SE, et al. Ten years of breast screening in the Nova Scotia Breast Screening Program, 1991-2001. Can Assoc Radiol J 2005;56:82-93.

44. Brekelmans CT, Seynaeve C, Bartels CC, et al. Effectiveness of breast cancer surveillance in BRCA1/2 gene mutation carriers and women with high familial risk. J Clin Oncol 2001;19:924-930.

45. Jassem J, Buchanan M, Janicke F, et al. The Hamburg statement: the partnership driving the European agenda on breast cancer. Eur J Cancer 2004;40:1810-1811.

46. Robson M, Gilewki T, Haas B, et al. BRCAassociated breast cancer in young women. J Clin Oncol 1998;16:1642-1649.

47. Tilanus-Linthorst MM, Kriege M, Boetes C, et al. Hereditary breast cancer growth rates and its impact on screening policy. Eur J Cancer 2005;41:1610-1617.
48. Chart PL, Franssen E. Management of women at increased risk for breast cancer: preliminary results from a new program. CMAJ 1997;157:1235-1242.

49. Lalloo F, Boggis CR, Evans DG, Shenton A, Threlfall AG, Howell A. Screening by mammography, women with a family history of breast cancer. Eur J Cancer 1998;34:937940.

50. Morris EA. Breast MRI for cancer screening in high-risk patients. Appl Radiol 2005; May(suppl):4-9. 